\title{
Stability determination for cyclized 2,4- dinitrophenyl hydrazone derivative of glucose
}

\author{
Muhammed Alzweiri ${ }^{1,2}$, Sara Al-Marabeh ${ }^{1,4^{*}}$, Sanaa K. Bardaweel ${ }^{1}$, Rawhi Alfar ${ }^{3}$ and Yusuf M. Al-Hiari ${ }^{1}$
}

\begin{abstract}
Background: The most well established tactic for the analysis of monosaccharaides, such as glucose, relies on derivatization procedures, using reagents as 2,4-dinitrophenylhydrazine (DNPH). Usually, the instability of the formed imine product deteriorates the detection of trace amounts of the sugar; rendering the spectrophotometric analysis of monosaccharaides extremely challenging.

Methods: In this study, we propose a modified derivatization procedure, reliant on the formation of a stable DNPHglucose derivative, to aid in the spectrophotometric analysis of glucose. The derivatization procedure was customized to perform the product work-up step under acidic conditions.

Results: The proton rich media resulted in direct reduction of the Schiff's base with concomitant intramolecular rearrangement of the product to yield a stable cyclized DNPH-glucose derivative. The annealed structure of the titled compound was verified by ${ }^{1} \mathrm{NMR},{ }^{13} \mathrm{C}-\mathrm{NMR}, \mathrm{HMBC}$ and $\mathrm{X}$-ray crystallography.

Conclusions: The derivative revealed extended stability in spiked plasma samples which suggests a potential to employ the described procedure for glucose analysis and detection in biological samples.
\end{abstract}

Keywords: 2,4-Dinitrophenyl hydrazine, Glucose, HPLC-UV, NMR and X-ray

\section{Background}

Monosaccharides are difficult to analyze because they have analogous physical and chemical characteristics (Medeiros and Simoneit 2007; Medlicott and Thompson 1985; Masuko et al. 2005). In addition, the lack of an appropriate chromophore makes the detection of monosaccharides via conventional UV-based instruments challenging (Dürr et al. 2004; Cheng and Her 2002). Several HPLC methods using various detection strategies were developed to assist the detection and analysis of monosaccharides (Castellari et al. 2000; Rogatsky et al. 2005). Nonetheless, low sensitivity and inapplicability to gradient elution hindered rapid and accurate determination of monosaccharides using these approaches (Ko et al. 2005).

Derivatization methods facilitate the chromatographic analysis of monosaccharides to sufficiently attain high

\footnotetext{
* Correspondence: s_marabeh@yahoo.com

${ }^{1}$ Department of Pharmaceutical Sciences, Faculty of Pharmacy, The University of Jordan, Amman 11942, Jordan

${ }^{4}$ Pharmaceutical Sciences Department, Faculty of Pharmacy, Zarqa University, Zarqa 13132, Jordan

Full list of author information is available at the end of the article
}

sensitivity in the very low concentration scale (Herbreteau 1992; Mopper and Johnson 1983). Numerous derivatizing reagents for monosaccharides have been reported in the literature (Evangelista et al. 1995; Chen F-T and Evangelista 1995; Strydom 1994), of which 2,4dinitrophenylhydrazine (DNPH) is the most widely used (Sanders and Schubert 1971; Siegel et al. 2009). Generally, DNPH has been frequently used as a derivatizing agent for carbonyl compounds, including reducing sugars for analytical purposes (Soman et al. 2008; Guan et al. 2012; Baños and Silva 2009), and it was reported to be used for derivatization of glucose (Gerees et al. 1962; Glaser and Zuckermann 1927). Through a condensation reaction, DNPH readily reacts with carbonyls in acidic conditions to form a 2,4-dinitrophenylhydrazone derivative. The reaction proceeds through a nucleophilic addition to the carbonyl moiety, followed by elimination of water to form hydrazone (Fig. 1). The resultant Schiff's base undergoes the reverse hydrolysis reaction, particularly in extreme $\mathrm{pH}$ conditions with the concurrent uptake of a water molecule (Barman 2014; Okano 


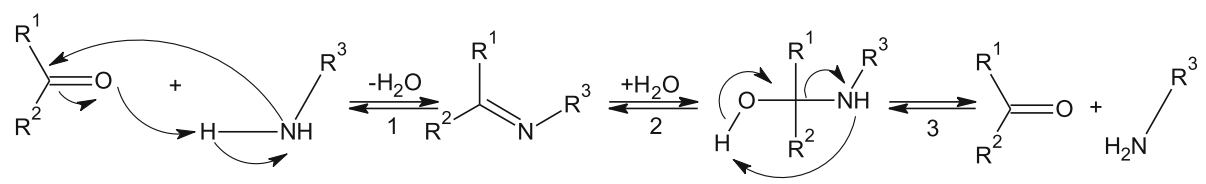

Fig. 1 Schiff's base formation and its hydrolysis

et al. 1980; Uchiyama et al. 2011). While the DNPH condensation reaction is direct at room temperature, the partial recovery of the formed Schiff's base limits the efficiency of the presumed derivatization procedure for sensitive analytical detection (Harvey 2011).

Recently, the quantitative measurement of monosaccharide glucose has captured a tremendous consideration in clinical laboratories due to its association to diabetes (Clarke et al. 1987; Clarke et al. 2005). The current glucose quantitative tests are generally based on enzymatic reactions that utilize glucose as a substrate (Heinemann 2010). Although such tests provide numerical quantitation of glucose in biological fluids, several studies demonstrated their lack of selectivity due to interactions with endogenous and exogenous substances other than glucose (e.g., maltose, ascorbic acid, acetaminophen, and icodextrin) (Heinemann 2010; Schleis 2007). In addition, temperature, humidity, and hematocrit were all shown to interfere with the precision and reliability of the currently used tests (Heller and Feldman 2008); hence, the demand for a selective, and yet, sensitive analytical procedure for glucose detection and quantitation is clear.

In the present study, the DNPH derivatization procedure of glucose was modified to include an acidic work-up step that resulted in the formation of a stable DNPH-glucose derivative (Fig. 2). In a manner that is analogous to the Amadori rearrangement, after the protonation of the formed Schiff base in the acidic work-up conditions, the reaction proceeds via spontaneous reaction to ultimately cause ring closure of the sugar moiety (Fig. 3). The structure of the resultant cyclic derivative was confirmed by means of NMR and X-ray crystallography. Subsequently, a stability-indicating HPLC method, with UV detection, was used for evaluating the product stability.

\section{Methods}

\section{Chemicals and reagents}

$\mathrm{D}_{6}$-dimethyl sulfoxide $99.9 \% \mathrm{D}, \mathrm{d}_{4}$-methanol, (+)-glucose, and glacial acetic acid were purchased from SigmaAldrich (St. Louis USA). 2,4-Dinitrophenylhydrazine, from the Laboratory Rasayan s.d.fine-chem LTD (Mumbai), was used in the derivatization reaction. Acetaldehyde, from VWR international Ltd (England), was used as internal standard. Absolute ethanol and ethyl acetate 99.5\%, purchased from Acros Organics (UK), was used in the product crystallization. Blank Plasma, used in the analysis, was donated from the University of Jordan Hospital. Methanol, analytical reagent grade, was purchased from lobachemie PVT. LTD (India), whereas HPLCgrade acetonitrile was purchased from the Anhui Fulltime specialized solvents \& reagents Co, LTD (China).

\section{Instruments}

\section{Chromatographic system}

Chromatographic analysis was fulfilled by using Shimadzu (Tokyo, Japan) HPLC system, model LC-20AT, with UV-detector SPD-20A, and equipped with LC20AT pump. The volume of the injector loop was $20 \mu \mathrm{l}$. Chromatographic separation was achieved by using C18, Thermo Hypersil-Keystone column, and mobile phase of acetonitrile and distilled water containing $10 \%$ glacial acetic acid (75: 25). The mobile phase flow rate was maintained at $0.2 \mathrm{ml} / \mathrm{min}$ during the run time. The wavelength of detection was set at $350 \mathrm{~nm}$ during the analysis.

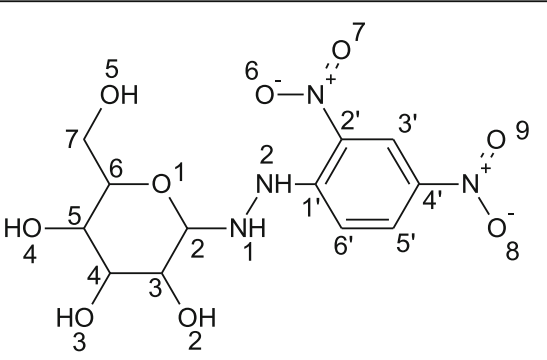

2-[2-(2,4-dinitrophenyl)hydrazinyl]-6-(hydroxymethyl)tetrahydro-2H-pyran-3,4.5-triol

Fig. 2 Structure and details of derivatized glucose product with 2,4-dinitrophenyl hydrazine (DNPH-glucose) 


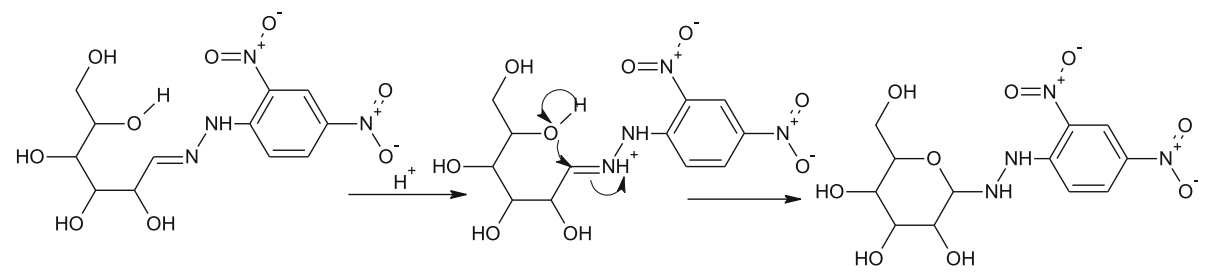

Fig. 3 Scheme of ring annealing of sugar in DNPH-glucose under acidic medium

\section{Nuclear magnetic resonance instrument}

One-dimensional and two-dimensional nuclear magnetic resonance spectra were recorded on Bruker Avance III NMR spectrometer $\left({ }^{1} \mathrm{H} 500.13 \mathrm{MHz},{ }^{13} \mathrm{C} 125.76 \mathrm{MHz}\right)$ controlled by Topspin 3.1 software. Samples of $20 \mathrm{mg}$ were dissolved in $\mathrm{d}_{4}$-methanol prior the analysis. Four transients of pulse sequence $\mathrm{Zg} 30$ were used to acquire ${ }^{1} \mathrm{H}$ spectra, 99 transients of Zgpg30 were used for acquiring ${ }^{13} \mathrm{C}$ data, and acquiring of heteronuclear multiple bond correlation (HMBC) was achieved. Temperature of the probe was maintained at $27{ }^{\circ} \mathrm{C}$ during the data acquisition. Chemical shifts were reported in parts per million related to tetramethylsilane (TMS) as internal standard. ${ }^{1} \mathrm{H}-\mathrm{NMR}$ of the product dissolved in $\mathrm{d}_{6}$-DMS was carried out by using NMR of Varian Oxford-300 (300 MHz) spectrometer.

\section{Data of nuclear magnetic resonance spectra for the DNPH- glucose}

$(\mathrm{A})^{1} \mathrm{H}-\mathrm{NMR} 500 \mathrm{MHz}$; MeOD: $\delta 3.27 \mathrm{ppm}(\mathrm{t}, J=8.9 \mathrm{~Hz}$, $1 \mathrm{H}, H 3), \delta 3.32 \mathrm{ppm}(\mathrm{m}, 2 \mathrm{H}, H 5$ and 6$), \delta 3.42 \mathrm{ppm}$ $(\mathrm{t}, J=8.8 \mathrm{~Hz}, 1 \mathrm{H}, H 4), \delta 3.72 \mathrm{ppm}(\mathrm{dd}, J=16.3$, $5.5 \mathrm{~Hz}, 1 \mathrm{H}, H 7 \mathrm{a}), \delta 3.93 \mathrm{ppm}(\mathrm{d}, J=11.55 \mathrm{~Hz}, 1 \mathrm{H}$, $H 7 b), \delta 4.07 \mathrm{ppm}(\mathrm{d}, J=8.9 \mathrm{~Hz}, 1 \mathrm{H}, H 2), \delta 8.05 \mathrm{ppm}$ $(\mathrm{d}, J=9.65 \mathrm{~Hz}, 1 \mathrm{H}, H 6)$ ), $\delta 8.29 \mathrm{ppm}(\mathrm{dd}, J=9.65$, $2.45 \mathrm{~Hz}, 1 \mathrm{H}, H 5)$, and $\delta 9.01 \mathrm{ppm}(\mathrm{d}, J=2.45 \mathrm{~Hz}, 1 \mathrm{H}$, $H 3)$. Additional peaks (for exchangeable protons) appeared by dissolving the product in DMSO: $\delta$ $3.12 \mathrm{ppm}$ (bs, $3 \mathrm{H}$ at 03,4 and 5$), \delta 6.23 \mathrm{ppm}(\mathrm{d}, J=$ $8.3 \mathrm{~Hz}, 1 \mathrm{H}$ at $\mathrm{O} 2), \delta 9.35 \mathrm{ppm}$ (bs, $1 \mathrm{H}$ at N1), and $\delta$ $9.65 \mathrm{ppm}$ (s, $1 \mathrm{H}$ at $\mathrm{N} 2)$.

(B) ${ }^{13}$ C-NMR $125 \mathrm{MHz}, \mathrm{MeOD}$ : ( $\left.\delta 61.37 \mathrm{ppm}, C 7\right),(\delta$ 70.13 ppm, C6), ( $\delta 71.34 \mathrm{ppm}, C 3),(\delta 77.56 \mathrm{ppm}, C 5)$, ( $\delta 77.64 \mathrm{ppm}, C 4),(\delta 90.55 \mathrm{ppm}, C 2),(\delta 116.38 \mathrm{ppm}$, C6), ( $\left.\delta 122.71 \mathrm{ppm}, C 3^{\prime}\right),\left(\delta 129.14 \mathrm{ppm}, C 5^{\prime}\right)$, $\left(\delta 129.21 \mathrm{ppm}, C 2^{\prime}\right),(\delta 136.51 \mathrm{ppm}, C 4)$, and ( $\delta 149.89 \mathrm{ppm}, \mathrm{C} 1$ ).

\section{$X$-ray single crystal structure determination}

A suitable yellow needle crystal with approximate dimensions $0.4627 \times 0.0408 \times 0.018 \mathrm{~mm}^{3}$ was Epoxy mounted on a glass fiber and the data collected at room temperature employing enhanced $\mathrm{Cu}$ radiation, $\lambda=1.54184 \AA$ and using Xcalibur/Oxford Diffractometer equipped with Eos CCD detector (Schleis 2007). CrysAlis Pro software was used for data collection, absorption correction, and data reduction (Schleis 2007): $3 \omega$ scan runs, 528 frames collected after optimization, exposure time $2-30 \mathrm{~s}, 1^{\circ}$ frame width, 45mm detector distance. Experimental absorption correction type is "multi-scan" with empirical absorption correction using spherical harmonics, implemented in SCALE3 ABSPACK scaling algorithm (Nowell et al. 2012) which was employed with min and max transmission factors of 0.946 and 0.980 , respectively (Additional file 1 ).

Cell parameters were retrieved using all observed reflections. The structure was originally solved using Olex2 (Dolomanov et al. 2009) as $\mathrm{P} 2{ }_{1}$ by direct methods and refined by least squares on $F^{2}$ to $R_{1}=0.0781[I \geq$ $2 \sigma(I)]$ with $\theta=3.25$ to $66.58^{\circ}$. Subsequent re-solution and refinement was done using SHELXTL program package (Xu et al. 2010) which reduced $R_{1}=0.0703$ with $\theta$ full $66.58^{\circ}$ and diffraction measurement fraction $\theta \max =0.982$. Goodness-of-fit on $F^{2}=1.061$. All nonhydrogen atoms were refined anisotropically with the hydrogen atoms placed constrained and assigned isotropic thermal parameters of 1.2 times that of the riding atoms. Largest diff. peak and hole were 0.465 and -0.450 e $\AA^{-3}$.

\section{Procedures}

The final product was prepared by dissolving a gram of glucose in a mixture of acetic acid $(3 \mathrm{ml})$, water $(2 \mathrm{ml})$, and methanol $(50 \mathrm{ml})$. Afterwards, an equimolar amount of 2,4dnitrophenylhydrazine ( $1.2 \mathrm{~g}$ ) was added to the reaction vessel and the mixture was refluxed for an hour. The vessel content was cooled and then poured onto acidified water (200 ml). Subsequently, the product was extracted three times by $10 \%$ ethanol in ethyl acetate $(100 \mathrm{ml}$ each) and dried by using anhydrous sodium sulfate. The organic layer was filtered and contracted by removing more than twothirds of the ethyl acetate content with aid of Rotavapor $\left(60{ }^{\circ} \mathrm{C}\right)$. The concentrated solution was left for $24 \mathrm{~h}$ to gradually precipitate, and product crystals were then filtered by a suction filter and allowed to stand to be completely dried over the bench. Retention factor (R.f) value was determined on TLC using solvent system of chloroform and methanol (8:2). The R.f value of resultant product was 0.48 compared with 0.88 of 2,4-dinitrophenyl hydrazine reactant. The melting point of product was $116-122^{\circ}$ which agrees with the 


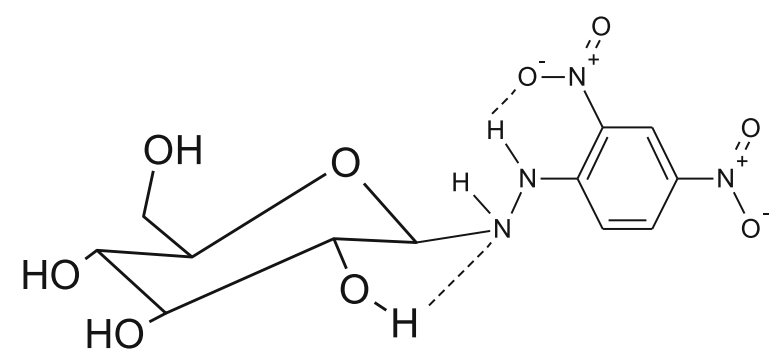

Fig. 4 Critical hydrogen bond formation in DNPH-glucose, extracted from ${ }^{1} \mathrm{H}$-NMR and $\mathrm{X}$-ray information, stabilizes the $\beta$-cyclic from of glucose part

reported melting point of $118-122^{\circ}$ (Gerees et al. 1962; Glaser and Zuckermann 1927). Solutions of $20 \mathrm{mg} \%$ were prepared from the product crystals in plasma $(\mathrm{pH}=7.3)$, basic (0.1 M NaOH, $\mathrm{pH}=13)$, acidic (0.1 M HCL, $\mathrm{pH}=1)$, and neutral media (water). Samples of $1 \mathrm{ml}$ were withdrawn from each solution each day and up to 10 days to study the stability of the product. Each sample was diluted five times with mobile phase prior to HPLC injection. The structure details of the product were characterized by NMR (1D and 2D) and X-ray analysis.

The derivatization procedure, used for the analysis of sugar content in plasma samples via HPLC, was fulfilled by mixing plasma $(50 \mu \mathrm{l})$ with the same volume of acetaldehyde solution $(25 \% \mathrm{w} / \mathrm{w})$ as internal standard. The contents were placed into an Eppendorf tube containing $1 \mathrm{ml}$ methanol and then vortex mixed and centrifuged. The liquor was mixed with $1 \mathrm{ml}$ of 2,4-dinitrophenylhydrazine solution (11\%) dissolved in acetic acid: methanol (15:100).
Subsequently, the sample was heated on a hotplate at $70^{\circ}$ for an hour. After cooling and filtration, $20 \mu \mathrm{l}$ of the sample was injected in HPLC instrument. The procedure was done in triplicate. The glucose solution (100 $\mathrm{mg} \%)$ was used as the standard and treated in the same manner as the sample.

\section{Results and discussion}

\section{Interpretation of ${ }^{1} \mathrm{H}-\mathrm{NMR}$ of the DNPH-glucose}

As illustrated in "Section Data of nuclear magnetic resonance spectra for the DNPH-glucose ," ${ }^{1} \mathrm{H}$-NMR spectra represents the structural details of the product (Fig. 2). Methylene hydrogens at carbon (7) were magnetically inequivalent; one of the hydrogens appears at $3.72 \mathrm{ppm}$ while the other appears at $3.93 \mathrm{ppm}$. This emphasizes a constrain of rotation for the methylene hydrogens and, consequently, suggests the cyclized form of the sugar part. Moreover, strong axial-axial coupling constants were clearly obtained between $\mathrm{H} 2$ / $\mathrm{H} 3, \mathrm{H} 3 / \mathrm{H} 4$, and $\mathrm{H} 4 / \mathrm{H} 5$. This further ensures the cyclic form of the sugar. Anomeric proton (2) that appears as a fine doublet at $4.07 \mathrm{ppm}$ with strong coupling constant $(8.9 \mathrm{ppm})$ clarifies the occurrence of the stable $\beta$ epimer of the sugar (Fig. 4).

Remarkably, the stability of the product is thought to be related to the imine reduction during the work-up of the reaction under acidic conditions, as depicted in Fig. 3. The reduction of imine bond was established by the appearance of broad singlet peak at $9.35 \mathrm{ppm}$ with integration of a single proton. Peak broadness is suggested to result from the free bond rotation that permits the formation of different sorts of hydrogen bonds and, hence the observed peak

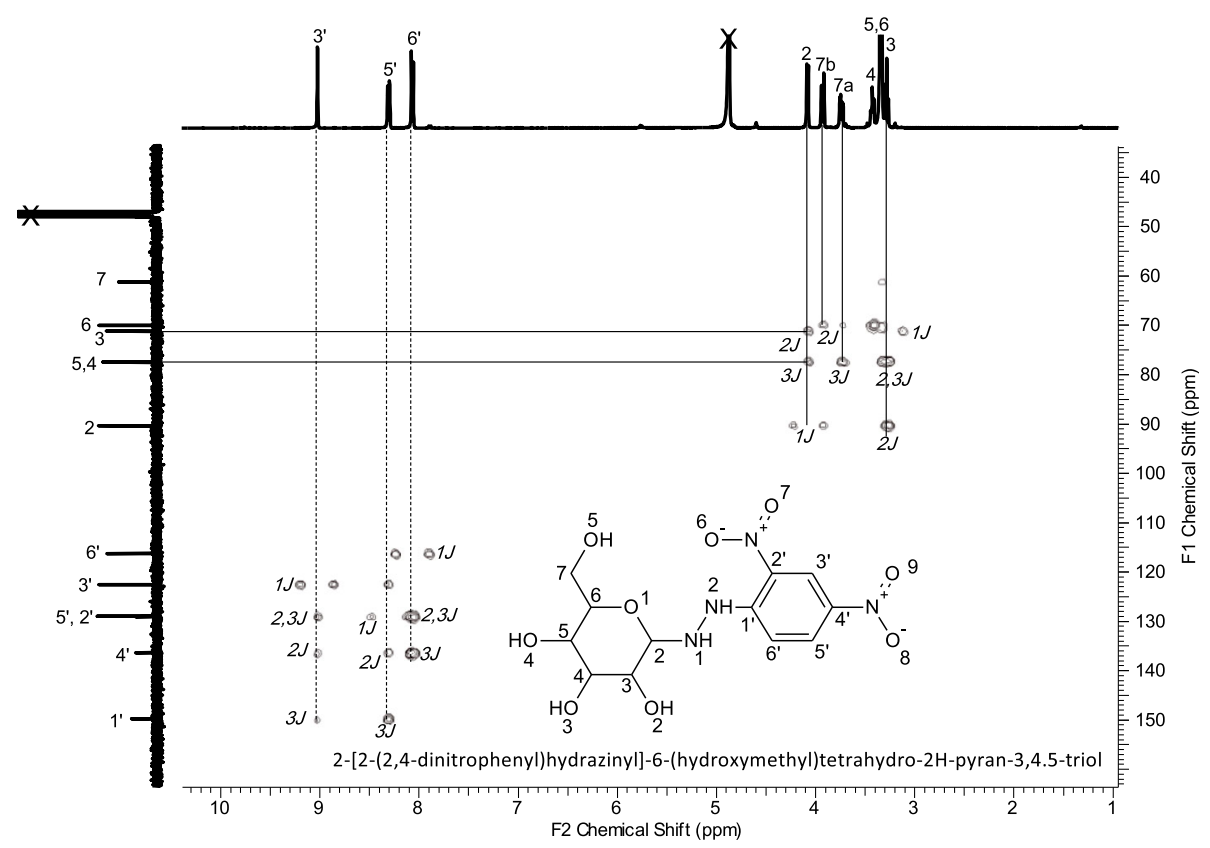

Fig. 5 Heteronuclear multiple bond correlation (HMBC) of DNPH-glucose 


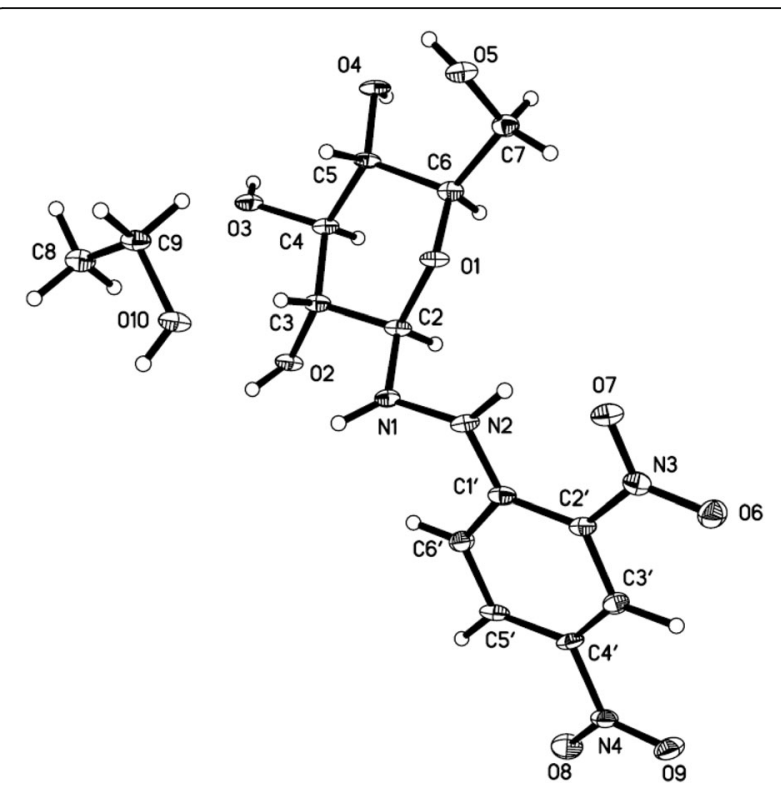

Fig. 6 Structure of DNPH-glucose obtained from single crystal x-ray analysis. Ethanol residue in the crystals resulted from hydrolyzed ethyl acetate used in product crystallization

broadening, or it might be due to coupling to $\mathrm{N}$ dipole. Interestingly, ${ }^{1} \mathrm{H}-\mathrm{NMR}$ spectrum in aprotic solvent (DMSO) showed a peak of hydroxyl hydrogen at $\mathrm{O} 2$ with a strong coupling to $\mathrm{H} 3(8.3 \mathrm{~Hz})$, which confirms the rigidity of hydroxyl proton. This might be attributed to its hydrogen bonding with N1. This is also confirmed by the deshielded effect on $\mathrm{O} 2$ hydrogen (6.23 ppm) in comparison with other hydroxyl hydrogens. Moreover, hydrogen on nitrogen (2) appears as the most deshielded peak with a sharp character due to a strong single type of hydrogen bonding with oxygen (6), as depicted in Fig. 4. Noteworthy, the chromophoric part of the structure (2,4-dinitrophenyl) remained stable during the reaction as the nitro groups were not reduced to amino groups. The lack of a signal at the aniline region in ${ }^{1} \mathrm{H}-\mathrm{NMR}$ of the titled compound, dissolved in DMSO, confirms the stability of nitro groups. Additionally, H3', characterized by its meta-coupling $(2.45 \mathrm{~Hz})$, appears as the most deshielded proton in ${ }^{1} \mathrm{H}-\mathrm{NMR} / \mathrm{MeOD}$. This cannot be obtained if the nitro group is reduced because amine is an electron-donating group by resonance effect whereas nitro is an electron withdrawing by resonance and inductive effects.

Interpretation of ${ }^{13} \mathrm{C}-\mathrm{NMR}$ and $\mathrm{HMBC}$ of the DNPH-glucose Carbon skeleton of the structure was confirmed by ${ }^{13} \mathrm{C}$ NMR, as illustrated in "Section Data of nuclear magnetic resonance spectra for the DNPH-glucose ." Carbon (2) was observed as the most deshielded carbon due to its binding with hydrazine and sugar groups. Although both carbons ( 2 ' and 4') are attached directly to nitro groups, and the mesomeric effect of hydrazine groups covers both of them,

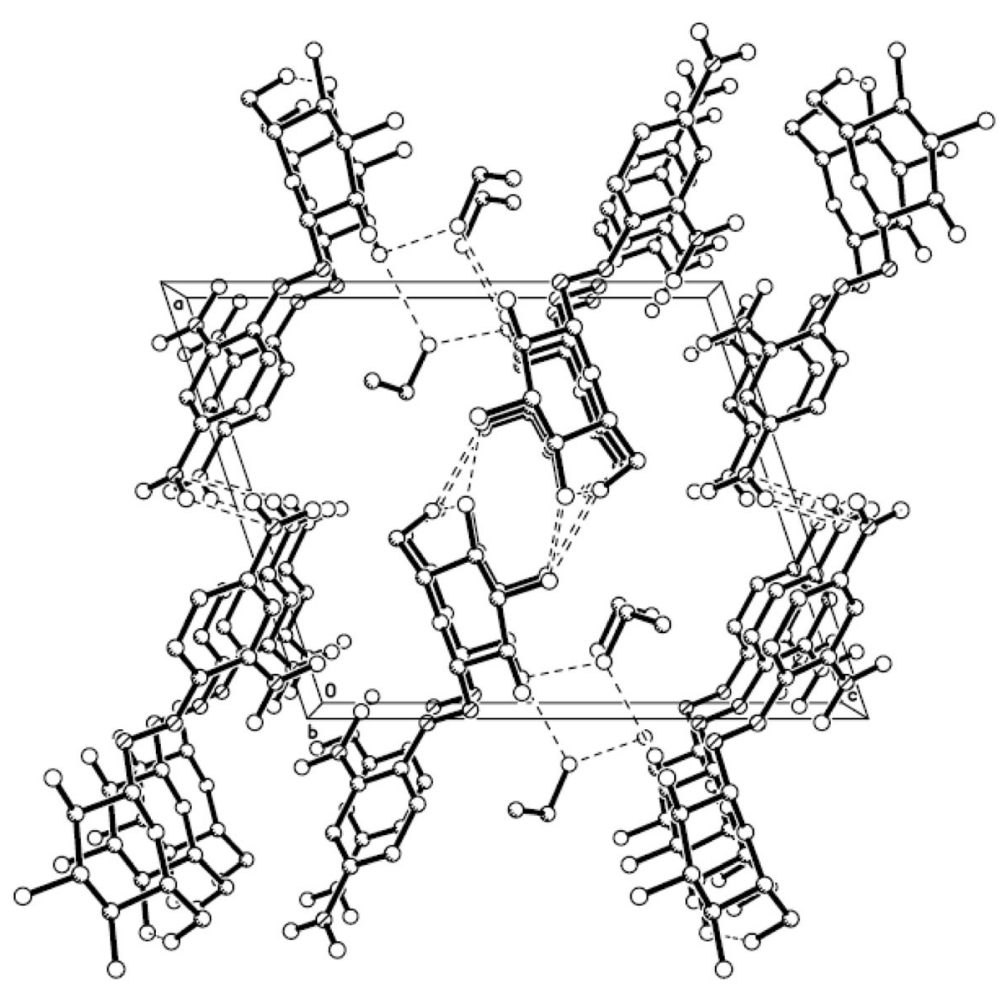

Fig. 7 Structure of DNPH-glucose crystal depicted the stacked layer of the structure enhanced by hydrogen bond formation 
carbon (4') has more deshielded signal (136.51 ppm) than carbon (2') which has a chemical shift of (129.21 ppm). This might be rationalized by the availability of nitro group at carbon (4') for inter-molecular interactions. The signals of carbon (4' and 2') were distinguished by the results obtained from HMBC correlation (Fig. 5). Hydrogen (5') has a 2Jcorrelation with the signal at $136.51 \mathrm{ppm}$ which should be 4'. Information of 1J-correlations between hydrogens and their corresponding carbons were also extracted from HMBC correlation chart. 1J-correlations were distinguished by two-point correlations as the correlation between carbon $\left(3^{\prime}\right)$ and its hydrogen.

\section{X-ray single crystal structure of DNPH-glucose}

Furthermore, the crystal structure of DNPH-glucose derivative has been determined by single crystal X-ray diffraction. The structure was solved by direct methods and refined to a final regression value of 0.07 . Molecular graphics and publication material were compiled using SHELXTL (Nowell et al. 2012). An Ortep drawing of the asymmetric unit (30\% probability) is given in Fig. 6. Intermolecular hydrogen bonds are observed between the glucose-hydrogens and ethanol oxygen (Figs. 6 and 7). The molecular packing, as illustrated in Fig. 7, shows that adjacent molecules are stacked on top of each other along the $b$ axis and demonstrates standard $\pi-\pi$ interactions between their exactly parallel (by symmetry) aryl planes.

\section{HPLC method for evaluating stability of DNPH-glucose}

Following the derivatization and the structural elucidation of the derivatized product, a stability-indicating method was used to assess the product stability. The applied method is capable of detecting the derivatized sugar based on its UV-active chromophore and was employed to evaluate the stability of the DNPH-glucose in different media. Peak identification was confirmed on the base of the retention times against an in-house standard injected individually through the HPLC system (Fig. 8).

Additionally, the proposed method was used to study the degradation kinetics profile of the DNPHglucose derivative under conditions representing acidic, basic, and neutral media (Fig. 9). The product appears stable in neutral and alkaline media, and more importantly, it is stable in spiked plasma samples. The
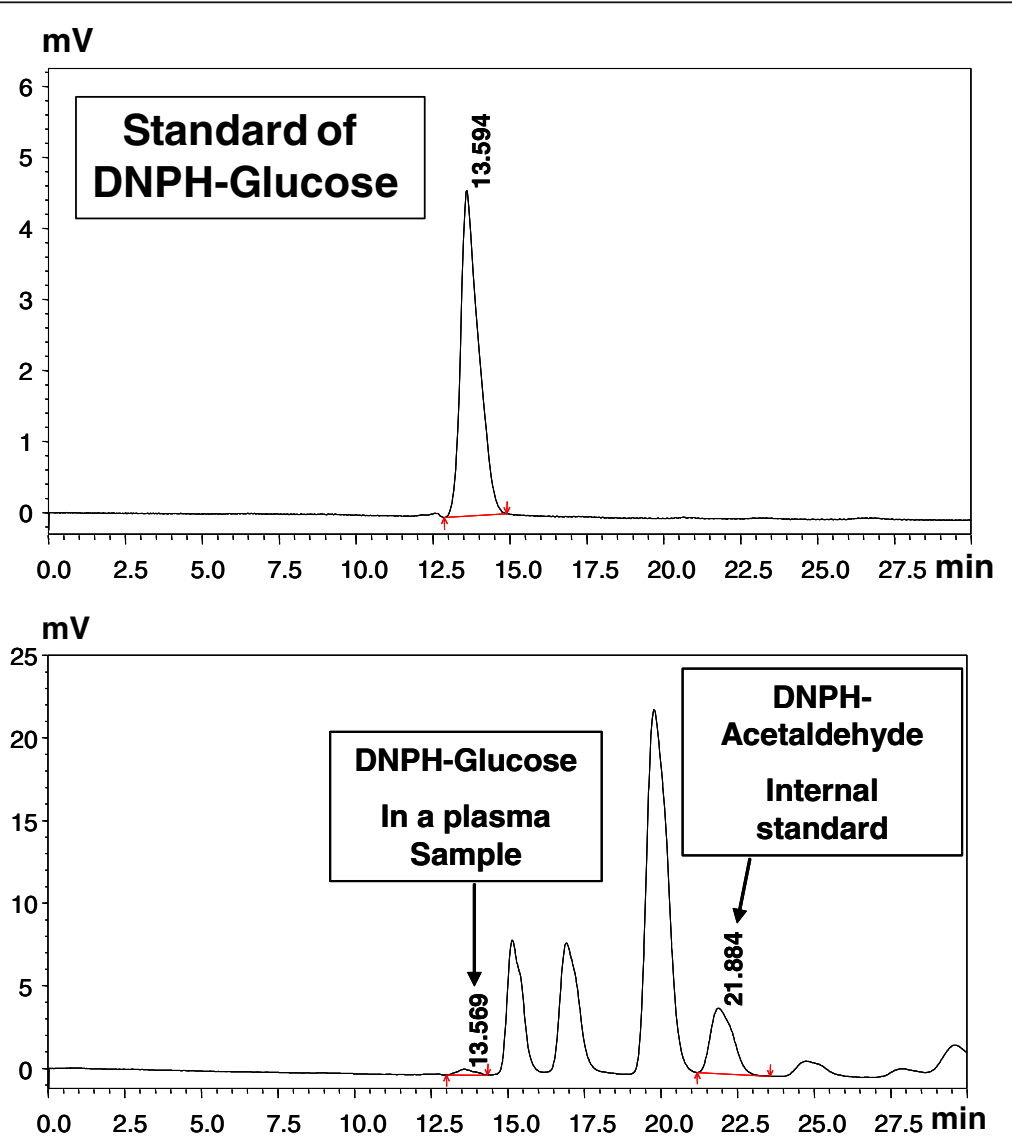

Fig. 8 Example of HPLC-UV chromatogram for standard DNPH-glucose and another for the derivatized sugar content in a plasma sample using acetaldehyde as internal standard to compensate possible losses during sample preparation 


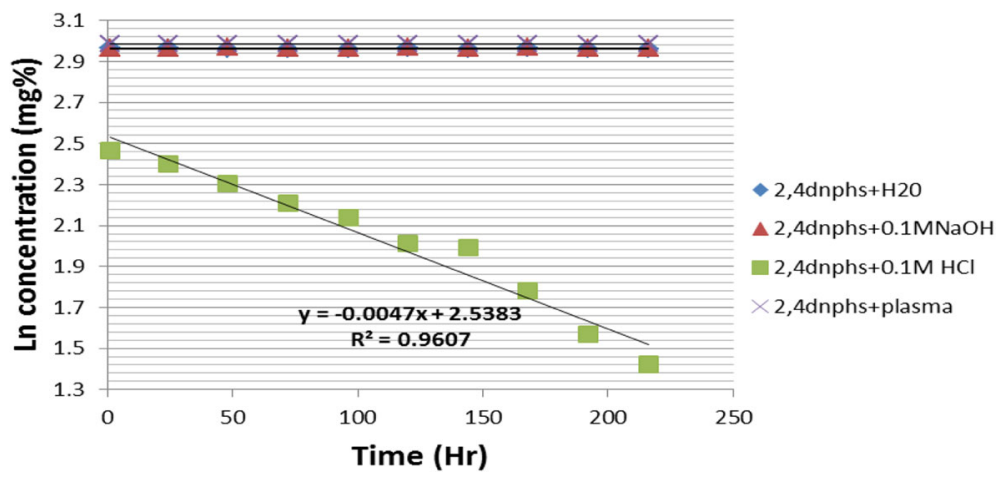

Fig. 9 Graph representing the degradation and stability of DNPH-glucose in several medium evaluated by HPLC-UV method

latter enables the use of this derivatization procedure for quantifying sugar content in plasma and ensures the stability of the samples during the analysis time, which does not usually exceed 10 days. Nevertheless, the observed product instability in acidic medium is most likely presumed due to the hydrolysis of the bond that links the sugar moiety to the chromophore in a mechanism resembling the hydrolysis of acetals and ketals (Fig. 10). The logarithmic transformation was used to obtain a linear behavior of product hydrolysis, and consequently, the degradation constant of the product in an acidic medium was calculated, Eq. 1.

$$
\operatorname{Ln} C=\operatorname{Ln} C^{0}-\mathrm{Kt}
$$

where $C$ is the concentration of remained product in the sample at each time point in $\mathrm{mg} \%$ unit and $C^{\mathrm{o}}$ stands for the initial concentration of the product in the sample (mg\%), whereas $t$ stands for the reaction time in hours.

The degradation kinetic of the product under acidic media follows typical first-order reaction kinetics as illustrated in (Fig. 10). Breakup of Schiff base is catalyzed by the presence of an acid and water. This occurs by acidic protonation of imine nitrogen, thus, making nearby carbon suitable for nucleophilic attack. Consequently, water molecule attacks imine carbon followed by proton transfer and elimination of amine (hydrazine) molecule. According to our results, the degradation rate of the derivatized sugar is $0.0047 \mathrm{~h}^{-1}$. i.e., just $1 \%$ of the product will be lost after each hour in acidic medium.

\section{Conclusions}

The structure of DNPH-glucose was verified with NMR and X-ray crystallography. NMR analysis of the DNPHglucose derivative confirms its cyclic sugar structure through the observed restriction of rotation for the methylene hydrogens. Besides, strong axial-axial coupling of the sugar hydrogens further fortifies the established cyclic structure of the sugar moiety. In addition, the X-ray structure of the DNPH-glucose derivative falls in good agreement with its NMR analysis in confirming the imine bond reduction and the formation of a stable hydrazine that is engaged in hydrogen bonds formation with the hydroxyl groups of the sugar. An extended stability of the DNPH-glucose derivative in spiked plasma samples was verified via the employed

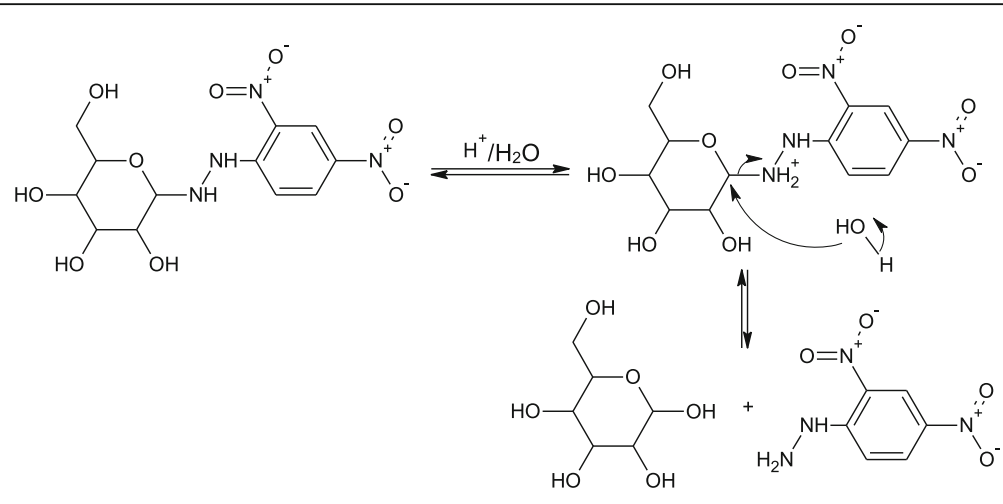

Fig. 10 Possible decomposition pathway of DNPH-glucose in acidic medium 
HPLC-UV method. The observed stability of the glucose derivative in the plasma keenly suggests a prospect for the described derivatization procedure to be employed in the spectrophotometric glucose analysis and detection in biological samples.

\section{Additional file}

Additional file 1: X-ray Data. (DOC $47 \mathrm{~kb}$ )

\section{Acknowledgements}

Not applicable

\section{Authors' contributions}

MA provided guidance, collected, and reviewed the literature and drafted the manuscript. SA performed experimental analytical work and helped in drafting the manuscript. SKB improved the quality of the manuscript. RA interpreted the X-ray data. YMA provided the synthesis chemistry work and helped in designing the manuscript. All authors read and approved final manuscript.

\section{Competing interests}

We wish to thank The University of Jordan represented by the Deanship of Academic Research for supporting and funding the project.

\section{Publisher's Note}

Springer Nature remains neutral with regard to jurisdictional claims in published maps and institutional affiliations.

\section{Author details}

${ }^{1}$ Department of Pharmaceutical Sciences, Faculty of Pharmacy, The University of Jordan, Amman 11942, Jordan. 'Faculty of Pharmacy, Al-Zaytoonah Private University of Jordan, Amman 11733, Jordan. ${ }^{3}$ Department of Physics, School of Science, Al-Balqa Applied University, Salt, Jordan. ${ }^{4}$ Pharmaceutical Sciences Department, Faculty of Pharmacy, Zarqa University, Zarqa 13132, Jordan.

\section{Received: 4 January 2017 Accepted: 4 April 2017}

\section{Published online: 28 April 2017}

\section{References}

Baños C-E, Silva M. In situ continuous derivatization/pre-concentration of carbonyl compounds with 2, 4-dinitrophenylhydrazine in aqueous samples by solid-phase extraction: application to liquid chromatography determination of aldehydes. Talanta. 2009;77(5):1597-602.

Barman BN. Accurate determination of aldehydes in amine catalysts or amines by 2, 4-dinitrophenylhydrazine derivatization. J Chromatogr A. 2014;1327:19-26.

Castellari M, Versari A, Spinabelli U, Galassi S, Amati A. An improved HPLC method for the analysis of organic acids, carbohydrates, and alcohols in grape musts and wines. J Liq Chromatogr Relat Technol. 2000; 23(13):2047-56.

Chen F-T A, Evangelista RA. Analysis of mono-and oligosaccharide isomers derivatized with 9-aminopyrene-1, 4, 6-trisulfonate by capillary electrophoresis with laser-induced fluorescence. Anal Biochem. 1995;230(2):273-80.

Cheng HL, Her GR. Determination of linkages of linear and branched oligosaccharides using closed-ring chromophore labeling and negative ion trap mass spectrometry. J Am Soc Mass Spectrom. 2002;13(11):1322-30.

Clarke WL, Cox D, Gonder-Frederick LA, Carter W, Pohl SL. Evaluating clinical accuracy of systems for self-monitoring of blood glucose. Diabetes Care. 1987;10(5):622-8.

Clarke WL, Anderson S, Farhy L, Breton M, Gonder-Frederick L, Cox D, Kovatchev B. Evaluating the clinical accuracy of two continuous glucose sensors using Continuous glucose-error grid analysis. Diabetes Care. 2005;28(10):2412-7.

Dolomanov OV, Bourhis LJ, Gildea RJ, Howard JAK, Puschmann H. OLEX2: a complete structure solution, refinement and analysis program. J Appl Cryst. 2009;42(2):339-41.
Dürr C, Hoffmeister D, Wohlert S-E, Ichinose K, Weber M, von Mulert U, Thorson JS, Bechthold A. The glycosyltransferase UrdGT2 catalyzes both C-and O-glycosidic sugar transfers. Angew Chem Int Ed. 2004;43(22):2962-5.

Evangelista RA, Liu M-S, Chen F-T A. Characterization of 9-aminopyrene-1, 4, 6-trisulfonate derivatized sugars by capillary electrophoresis with laserinduced fluorescence detection. Anal Chem. 1995;67(13):2239-45.

Gerees A, Somogyi L, Konya A, Bukovecz M. Conversion of some aldose 2,4-dinitrophenylhydrazones. Acta Chim Acad Sci Hung. 1962;30:95-103.

Glaser E, Zuckermann N. Hydrazine compounds of a-glucoheptose. Z Physiol Chem. 1927;167:37-69.

Guan X, Rubin E, Anni H. An optimized method for the measurement of acetaldehyde by high-performance liquid Chromatography. Alcohol Clin Exp Res. 2012;36(3):398-405

Harvey DJ. Derivatization of carbohydrates for analysis by chromatography; electrophoresis and mass spectrometry. J Chromatogr B. 2011:879(17):1196-225.

Heinemann L. Quality of glucose measurement with blood glucose meters at the point-of-care: relevance of interfering factors. Diabetes Technology and Therapeutics. 2010;12(11):847-57.

Heller A, Feldman B. Electrochemical glucose sensors and their applications in diabetes management. Chem Rev. 2008;108(7):2482-505.

Herbreteau B. Review and state of sugar analysis by high performance liquid chromatography. Analusis. 1992;20(7):355-74

Ko JH, Huang H, Kang GW, Cheong WJ. Simultaneous quantitative determination of monosaccharides including fructose in hydrolysates of yogurt and orange juice products by derivatization of monosaccharides with $\mathrm{p}$-aminobenzoic acid ethyl ester followed by HPLC. Bulletin-Korean Chemical Society. 2005;26(10):1533

Masuko T, Minami A, Iwasaki N, Majima T, Nishimura S-I, Lee YC. Carbohydrate analysis by a phenol-sulfuric acid method in microplate format. Anal Biochem. 2005;339(1):69-72.

Medeiros PM, Simoneit BRT. Analysis of sugars in environmental samples by gas chromatography-mass spectrometry. J Chromatogr A. 2007;1141(2):271-8.

Medlicott AP, Thompson AK. Analysis of sugars and organic acids in ripening mango fruits (Mangifera indica L. var Keitt) by high performance liquid chromatography. J Sci Food Agric. 1985;36(7):561-6.

Mopper K, Johnson L. Reversed-phase liquid chromatographic analysis of dnssugars: Optimization of derivatization and chromatographic procedures and applications to natural samples. J Chromatogr A. 1983;256:27-38.

Nowell H, Barnett SA, Christensen KE, Teat SJ, Allan DR. 119, the small-molecule single-crystal diffraction beamline at Diamond Light Source. J Synchrotron Radiat. 2012;19(3):435-41.

Okano V, Bastos MP, Do Amaral L. Kinetics and mechanism for pyridine $\mathrm{N}$-oxide carboxaldehyde phenylhydrazone formation. J Am Chem Soc. 1980;102(12):4155-9.

Rogatsky E, Jayatillake H, Goswami G, Tomuta V, Stein D. Sensitive LC MS quantitative analysis of carbohydrates by $\mathrm{Cs}<$ sup $>+</$ sup $>$ Attachment. J Am Soc Mass Spectrom. 2005;16(11):1805-11.

Sanders EB, Schubert J. Spectrophotometric analysis of carbonyl compounds in the presence of carbohydrates without prior separation. Anal Chem. 1971; 43(1):59-62.

Schleis TG. Interference of maltose, icodextrin, galactose, or xylose with some blood glucose monitoring systems. Pharmacotherapy: The Journal of Human Pharmacology and Drug Therapy. 2007;27(9):1313-21.

Siegel D, Rasenko T, Koch M, Nehls I. Determination of the $<\mathrm{i}>$ Alternaria $</ \mathrm{i}>$ mycotoxin tenuazonic acid in cereals by high-performance liquid chromatography-electrospray ionization ion-trap multistage mass spectrometry after derivatization with 2, 4-dinitrophenylhydrazine. J Chromatogr A. 2009;1216(21):4582-8.

Soman A, Qiu Y, Li QC. HPLC-UV method development and validation for the determination of low level formaldehyde in a drug substance. J Chromatogr Sci. 2008:46(6):461-5.

Strydom DJ. Chromatographic separation of 1-phenyl-3-methyl-5-pyrazolonederivatized neutral, acidic and basic aldoses. J Chromatogr A. 1994; 678(1):17-23.

Uchiyama S, Inaba Y, Kunugita N. Derivatization of carbonyl compounds with 2 4-dinitrophenylhydrazine and their subsequent determination by highperformance liquid chromatography. J Chromatogr B. 2011;879(17):1282-9.

Xu J-J, Zhu Z, Shi S-M, Zhou J, Jin C-M. Syntheses and crystal structures of two cadmium coordination polymers [Cd (2-mBIM)(NCS)(SCN)] $n$ and $[\mathrm{Cd} 2$ (2-mBIM) 2 (NO3) 2 (C4H4O4)(H2O) 5] n based on bis (2-methylimidazol-1-yl) methane. J Coord Chem. 2010;63(13):2296-306. 